\title{
Feasibility of using Lokomat combined with functional electrical stimulation for the rehabilitation of foot drop
}

\author{
Christian B. Laursen (1), Jørgen F. Nielsen (2), Ole K. Andersen (1), Erika G. Spaich (1)
}

(1) Integrative Neuroscience group, SMI®, Department of Health Science and Technology, Aalborg University, Denmark. (2) Hammel Neurorehabilitation and Research Centre, Aarhus University, Denmark

This article is distributed under the terms of the Creative Commons Attribution Noncommercial License (CC BY-NC 4.0) which permits any noncommercial use, distribution, and reproduction in any medium, provided the original author(s) and source are credited.

\begin{abstract}
This study investigated the clinical feasibility of combining the electromechanical gait trainer Lokomat with functional electrical therapy (LokoFET), stimulating the common peroneal nerve during the swing phase of the gait cycle to correct foot drop as an integrated part of gait therapy. Five patients with different acquired brain injuries trained with LokoFET 2-3 times a week for 3-4 weeks. Pre- and post-intervention evaluations were performed to quantify neurophysiological changes related to the patients' foot drop impairment during the swing phase of the gait cycle. A semi-structured interview was used to investigate the therapists' acceptance of LokoFET in clinical practice. The patients showed a significant increase in the level of activation of the tibialis anterior muscle and the maximal dorsiflexion during the swing phase, when comparing the pre- and post-intervention evaluations. This showed an improvement of function related to the foot drop impairment. The interview revealed that the therapists perceived the combined system as a useful tool in the rehabilitation of gait. However, lack of muscle selectivity relating to the FES element of LokoFET was assessed to be critical for acceptance in clinical practice.

Keywords: Automation, functional electrical stimulation (FES), neuromuscular stimulation, gait, robot
\end{abstract}

Eur J Transl Myol 201626 (3) 268-273

Over a third of the patients suffering from acquired brain injury (ABI) have gait impairments. ${ }^{1,2}$ An inadequate control of dorsiflexion during the swing phase of gait (called foot drop) is often seen. ${ }^{3}$ Foot drop is often caused by weakness of the tibialis anterior (TA) muscle, ${ }^{4}$ which can result in compensatory movement patterns, ${ }^{3}$ slowed gait velocity, ${ }^{5}$ limited functional mobility, and increased risk of falls. ${ }^{4}$ Electromechanical gait trainers (EMGT) such as Lokomat (Hocoma AG, Volketwill, Switzerland) are often utilized to initiate early intensive gait rehabilitation. ${ }^{6}$ Lokomat enables the patient to train gait movements with many repetitions, more independently of the therapist, potentially with their body weight supported, and with an automated movement of the lower extremities. ${ }^{6}$ Stroke patients subjected to EMGT therapy (using Lokomat) combined with conventional therapy developed larger muscle mass and lower fat percentage compared to controls only receiving conventional therapy. ${ }^{7}$ Despite the beneficial effects of this EMGT therapy, Lokomat has shown to inhibit dorsiflexion of the ankle joint during the swing phase of the gait cycle, rather than facilitating it. $^{8}$ This conflicts with the guidelines for rehabilitation of pathological gait, which advocate that rehabilitation should facilitate a physiological gait pattern, supporting restorative neurorehabilitation. ${ }^{3}$

Functional electrical stimulation (FES) administered at the common peroneal nerve has shown to facilitate dorsiflexion of the ankle joint during the swing phase of the gait cycle, ${ }^{9}$ and there is clinical evidence supporting FES having a therapeutic effect on the foot drop impairment. ${ }^{10}$ The combination of EMGT and FES for rehabilitation of gait impairments in sub-acute stroke patients has resulted in increased gait speed compared to conventional therapy. ${ }^{11}$ However, only two studies have combined FES with Lokomat, showing that the combined system was technically feasible, ${ }^{12}$ and that when tested by a stroke patient, the patient was able to achieve better dorsiflexion and TA muscle activation during the swing phase of the gait cycle, in comparison to the normal gait pattern. ${ }^{13}$ The goal of this feasibility study was therefore to test a clinical protocol combining Lokomat with Functional Electrical Therapy (FET), i.e. LokoFET, as preparation for larger clinical trials. 


\section{Lokomat and FES for foot drop}

Eur J Transl Myol 26 (3) 268-273

The aims of the study were to:

- quantify the neurophysiological changes related to the patient's foot drop impairment after LokoFET treatment;

- investigate the therapists' acceptance of LokoFET in clinical practice.

\section{Materials ans Methods}

Test of LokoFET in clinical practice

\section{Subjects}

Five patients were recruited (Table 1) based on the following inclusion criteria: suffering from $\mathrm{ABI}$, age between $18-80$ years, weight $50-100 \mathrm{~kg}$, femur length $48-21 \mathrm{~cm}$, able to fully extend the knees, able to communicate and understand instructions, tolerate electric stimulation, tolerate to be supported passively in the body weight support (BWS) harness for $5 \mathrm{~min}$, decreased dorsiflexion during the swing phase, and able to walk a minimum of 30 steps with the ankle strap from Lokomat in neutral position. Patients with the following criteria were excluded: height over $2 \mathrm{~m}$, leg length difference larger than $2 \mathrm{~cm}$, bone instability, infection and/or orthopedic problems in the placement area of the electrodes, heart or lung disease, pregnancy, pacemaker, prior incidences of neurological or musculoskeletal diseases, suffering from mental diseases, and lack of cooperation. The protocol for the study was approved by the local ethical committee (ESDH 1-10-72-135-12) and the experiments were conducted in accordance with the declaration of Helsinki.

\section{Lokomat}

Lokomat was used to provide gait training. Prior to the first experimental session, the patients had at least one training session in Lokomat to familiarize them to the EMGT. At each session, the Lokomat exoskeleton, gait speed, and BWS were adjusted to fit the patient. The ankle straps were adjusted to have the patient's ankle joint in neutral position. When the gait training was initiated, Lokomat supplied timing information, defining the start and end of the stance phases.

Functional electrical stimulation

To stimulate the common peroneal nerve, a one-

Table 1. Demographic data of patients

\begin{tabular}{|c|c|c|c|c|c|}
\hline Patient & 1 & 2 & 3 & 4 & 5 \\
\hline Age & 18 & 26 & 18 & 64 & 32 \\
\hline $\begin{array}{l}\text { Days since } \\
\text { ABI }\end{array}$ & 108 & 106 & 108 & 25 & 76 \\
\hline $\begin{array}{l}\text { Affected body } \\
\text { side }\end{array}$ & Left & Right & Right & Right & Right \\
\hline Type of ABI & TBI & TBI & TBI & Stroke & TBI \\
\hline
\end{tabular}

TBI: traumatic brain injury; ABI: acquired brain injury channel, computer-controlled stimulator (Noxitest, Danmark) and two surface electrodes were used. The cathode (Pals Platinum Round $3.2 \mathrm{~cm}$, Axelgaard Ltd., USA) was placed above the common peroneal nerve, close to the fibular head on the patient's most affected leg. The anode (Pals Platinum Oval 4.0x6.4 cm, Axelgaard Ltd., USA) was placed on the proximal aspect of the TA muscle on the same leg. The rectangular monophasic stimulation had a pulse duration of $300 \mu \mathrm{s}$ and a frequency of $30 \mathrm{~Hz}$. Adjustments regarding the location of the electrodes and the stimulation intensity were done in each session, based on the evoked motor response. FES was triggered using custom made software. ${ }^{13}$ The duration of the stimulation period was controlled by the output signals from Lokomat. The start signal corresponded to heel-strike of the contralateral leg and the end signal corresponded to the beginning of the stance phase of the most affected leg. ${ }^{13}$ Consequently, the patient's most affected side was stimulated during the push-off and swing phases of the gait cycle.

\section{Outcome measurements}

Electromyography (EMG) was recorded using a bipolar configuration. Two electrodes (Medicotest, Oelstykke, Denmark) were placed on the muscle belly of TA on the patient's most affected leg and a third electrode of the same kind was placed on the tibial bone serving as reference. The recordings were amplified, based on the patient's individual maximum voluntary contraction, band-pass filtered (10-500 Hz, second order), sampled at $2 \mathrm{kHz}$, and saved. Kinematics of the ankle joint were recorded in the sagittal plane using an electronic goniometer (SG110/A, Biometrics Ltd., Gwent, UK). The goniometer was placed on the lateral side of the ankle of the most affected leg. Data was sampled at 2 $\mathrm{kHz}$ and saved. The output timing signals from Lokomat were also sampled at $2 \mathrm{kHz}$, saved, and used to detect the phases of the gait cycle in the later offline analysis.

Pre- and post-intervention evaluation

Pre- and post-intervention evaluations were performed to assess the effect of training. The goniometer and EMG electrodes were mounted while the patients were seated. The patients then walked in Lokomat for 2-5 minutes before recording the ankle kinematics and TA EMG during 30 steps.

\section{LokoFET training}

Patients had one hour set aside per session. First, the FES stimulation intensity was determined starting at $10 \mathrm{~mA}$ and adding steps of $2 \mathrm{~mA}$ until adequate dorsiflexion was observed $\left(\geq 10^{\circ}\right)$ with the patient in sitting position. The Lokomat's BWS system, exoskeleton, and treadmill were then adjusted. Immediately afterwards, the patients walked assisted by Lokomat and, if necessary, the stimulation intensity was adjusted until appropriate dorsiflexion was visually 


\section{Lokomat and FES for foot drop}

Eur J Transl Myol 26 (3) 268-273

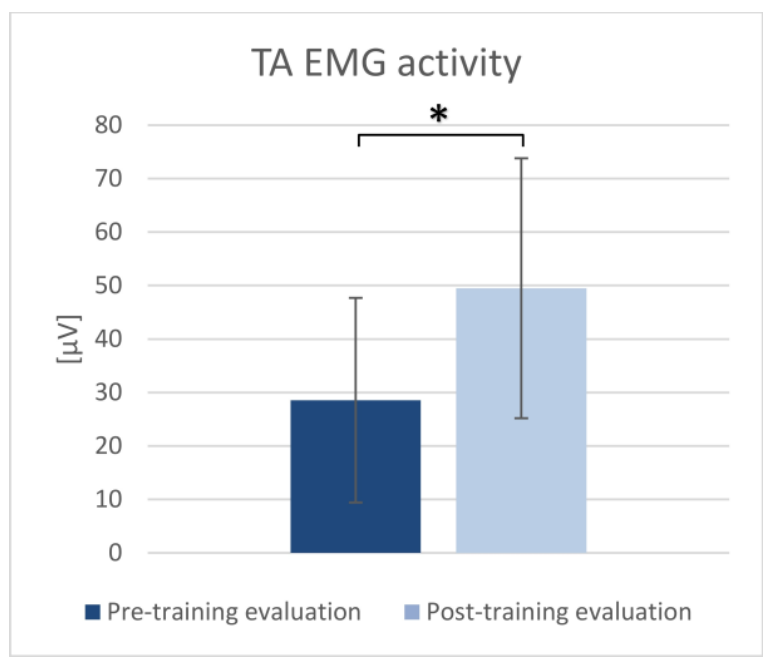

Fig 1. TA EMG activity measured during the pre- and post-training evaluations.

confirmed, or the stimulation intensity became intolerable. The patients received LokoFET training 2-3 times a week for 3-4 weeks, giving a total of 6-8 treatments. The time spent on preparing for FES (e.g. placement of electrodes and adjusting stimulation intensity) before the actual training started was noted. The time spent on preparing Lokomat's BWS and exoskeleton, and setting the speed of the treadmill was likewise noted.

\section{Data analysis}

The TA EMG activity in each step was assessed by the RMS in a window comprising the push-off and swing phases of the most affected leg. The RMS values corresponding to the individual steps in each session were then averaged. The kinematics of the ankle joint was assessed as the maximal angle variation in the same window as the EMG, but divided in two intervals due to the biphasic nature of the movement of the ankle joint. To assess the maximal plantarflexion, the window started at push-off and ended at the point of peak plantarflexion, indicating the end of the push-off phase. To assess maximal dorsiflexion, the window started at the point of peak plantarflexion and ended at heelcontact.

\section{Statistical analysis}

Data were analyzed using a two-tailed paired t-test having time as the factor (pre- and post-intervention evaluation). If data was not normally distributed, a Wilcoxon signed rank test was performed. A significance level of $\mathrm{p}<0.05$ was used.

Acceptance of LokoFET in clinical practice

To investigate the acceptance of LokoFET in clinical practice a semi-structured interview was performed and data was saved as transcripts ${ }^{14}$. The respondents for the semi-structured interview were two of the physiotherapists who administered LokoFET. The analysis of the qualitative data was done using a conventional qualitative content analysis. ${ }^{15}$ After

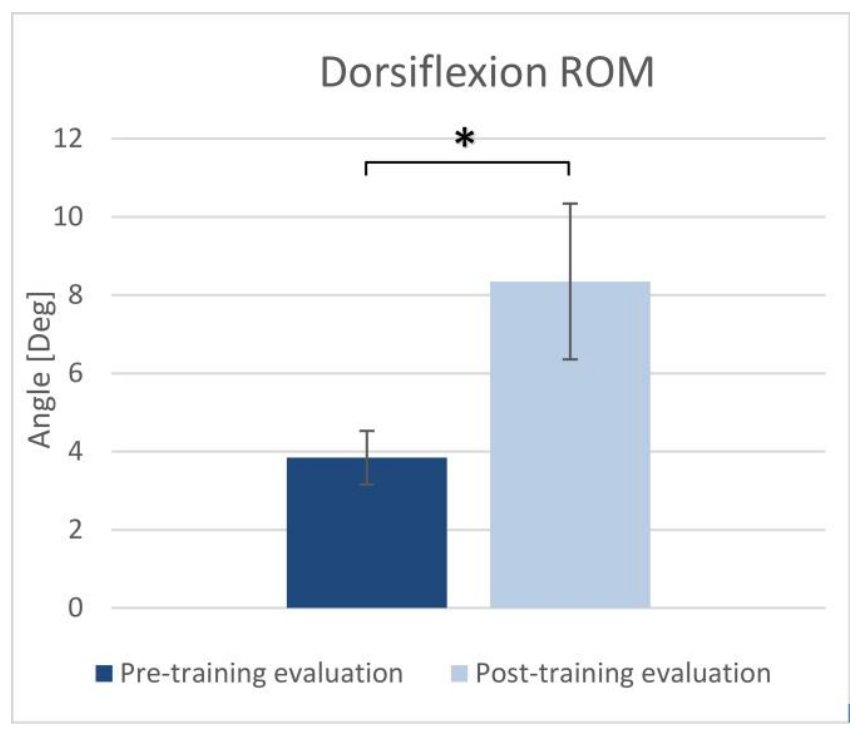

Fig 2. Maximal dorsiflexion measured during the pre-and post-training evaluations. 


\section{Lokomat and FES for foot drop}

Eur J Transl Myol 26 (3) 268-273

reading the transcripts, the material was coded allowing for emerging themes to appear as sub-themes, resulting in a meaning condensation, and interpretation of the qualitative data. ${ }^{15}$

\section{Results}

Test of LokoFET in clinical practice

Out of the five patients originally included in the study, four patients completed the LokoFET training course. Patient 5 was excluded due to low motor ability and low cognitive stamina making him unable to complete the LokoFET training within the one-hour limit per session. The mean stimulation intensity across patients was $34.98 \pm 8.25 \mathrm{~mA}$. The mean duration of the sessions was $17.12 \pm 6.34$ minutes. $10.35 \pm 3.83$ and $35.91 \pm 6.00$ minutes were spent for FES and Lokomat preparation, respectively.

\section{Effect of LokoFET training}

The TA EMG activity was significantly increased $(73.34 \%)$ at the post-intervention evaluation, compared to the pre- intervention evaluation (paired t-test, $\mathrm{p}<0.05$ ) (Figure 1). The maximal dorsiflexion during the swing phase was significantly increased $(117.39 \%)$ at the postintervention evaluation, compared to the preintervention evaluation (paired t-test, $\mathrm{p}<0.02$ ) (Figure 2 ). The maximal plantarflexion during push-off was not changed at the post-intervention evaluation, compared to the pre-intervention evaluation (paired t-test, $\mathrm{p}=0.67$ ) (Figure 3).

Acceptance of LokoFET in clinical practice

The therapists mentioned the FES element as a positive and active add-on treatment technique to the conventional EMGT therapy. However, it was also observed that in some LokoFET training sessions, FES resulted in dorsiflexion coupled with eversion of the ankle joint. Selective dorsiflexion was assessed by the therapists to be crucial for the acceptance of LokoFET in clinical practice. This is because the coupled dorsiflexion and eversion is part of the pathological gait pattern, which the therapists stride towards avoiding during gait training, especially during the sub-acute phase. Training in the application and use of FES for foot drop correction were therefore assessed to be crucial by the therapists. Time spent on FES preparation prolonged the usual time spent on preparation before training with Lokomat. The therapists argued that this could potentially harm the usefulness of LokoFET because many patients suffer from fatigue, hereby requiring a short preparation time in order to train before fatigue sets in. The therapists also argued that the mere use of Lokomat in the standard EMGT therapy (without FES) was highly complex on its own, especially when training heavily motor impaired patients. The introduction of FES could therefore add a level of complexity making them unable to use both Lokomat and FES.

\section{Discussion}

Test of LokoFET in clinical practice

It was feasible for patients with different $\mathrm{ABI}$ to complete the LokoFET training in a clinical environment. The British Medical Research Council highlights the importance of feasibility studies evaluating the practical feasibility when introducing new interventions in everyday practice before testing them in larger clinical trials. ${ }^{16}$ This study did not aim to show the effectiveness of LokoFET compared to conventional EMGT therapy or spontaneous remission. However, when comparing the pre- and postintervention evaluations, both the TA EMG activity and the ankle joint kinematics (dorsiflexion) showed significant gains related to the foot drop impairment. In this study, the stimulation intensity was adjusted until appropriate dorsiflexion was visually confirmed, or the stimulation intensity became intolerable. Alternatively, the adjustment of the stimulation intensity could have been based on more precise kinematic information using electronic goniometers or other sensors external to the

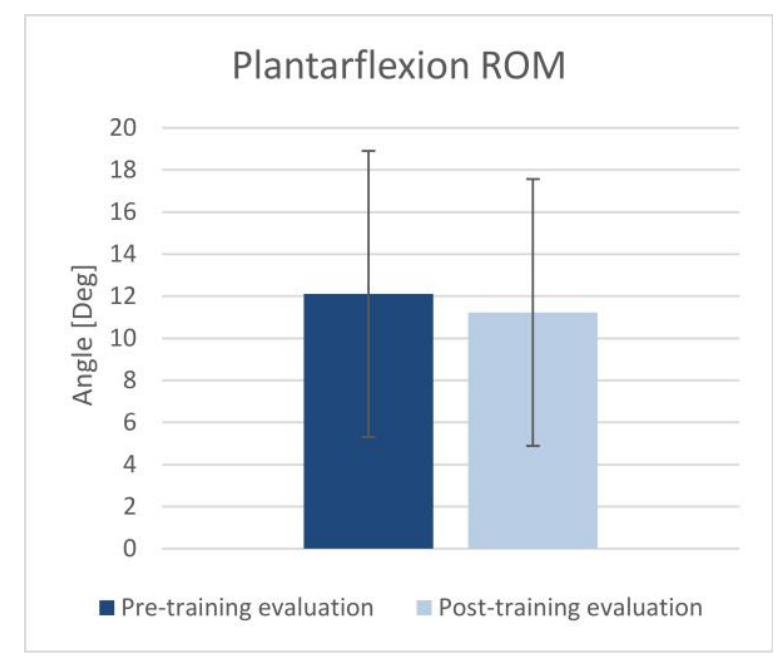

Fig 3. Maximal plantarflexion measured during the pre-and post-training evaluations. 


\section{Lokomat and FES for foot drop}

Eur J Transl Myol 26 (3) 268-273

Lokomat. However, one of the goals of this FES add-on solution was to be as simple to utilize as possible. The use of an external sensor would therefore have increased the complexity of the system. Weakness of ankle dorsiflexors is a known cause of foot drop. ${ }^{4}$ Furthermore, reduced strength of the dorsiflexors has shown to be a primary determinant for slowed gait velocity and temporal asymmetry in stroke patients. ${ }^{17}$ Increased TA EMG activity and dorsiflexion, as observed in this study, can therefore be interpreted as signs of recovery. The rational for combining Lokomat and FET was to provide with more active training of the ankle joint in comparison to traditional Lokomat training. Active, highly repetitive, and task-specific training has been shown important in creating better neurological rehabilitation outcomes. ${ }^{18}$ LokoFET seemed to provide the conventional EMGT therapy with more active ankle joint training compared to EMGT alone, as in Spaich et al. 2014. ${ }^{13}$ Additionally to the inhibition of dorsiflexion caused by the EMGT used in this study, the movement of the hip joint is restricted to the sagittal plane, ${ }^{8}$ which does not fully support a physiological gait movement. Future studies might benefit on using an EMGT, which allows both adduction/abduction and rotation of the hip joint. The LokoFET system uses a treadmill to provide gait therapy. A similar EMGT system using a robot-driven exoskeleton orthosis and over-ground walking could have been used instead. ${ }^{6}$ However, over-ground walking might demand a better control of balance from the patient, leaving patients with severely impaired balance unable to perform this kind of therapy. When comparing normal over-ground walking with treadmill walking, treadmill walking shows higher cadence, shorter swing phase, shorter step length and longer stance phase with a lower level of hip extension. ${ }^{19}$ Treadmill walking is therefore somewhat different compared to over-ground walking, which must be considered the norm. On the other hand, treadmill training allows for training in a small stationary environment, with a high level of control, making it easy to use measuring, support, and visual biofeedback equipment, ${ }^{19}$ which was desirable in the present case. To further evaluate the effectiveness of LokoFET, a larger clinical trial would be necessary. The current study has presented a feasible training protocol and provided grounds for sample size calculations for future clinical trials.

\section{Acceptance of LokoFET in clinical practice}

The FES element of LokoFET was found useful as an add-on treatment technique in clinical practice, as a tool to provide the patients with active training of their foot drop. Perceived usefulness in the eyes of the healthcare personnel is a critical aspect for obtaining acceptance of a new technology. ${ }^{20}$ The fact that the therapists found the FES element useful provides good basis for acceptance of LokoFET in clinical practice. On the other hand, the observed eversion coupled with the desired dorsiflexion, impacted negatively on the perception of usefulness of LokoFET. This aspect was assessed to be critical, in order for LokoFET to be useful in clinical practice. Changing the stimulation location and reducing the stimulation intensity, could in many cases diminish the undesired eversion and more selective dorsiflexion could be produced. The use of electrode arrays with multiple active stimulation channels might in this context ease the identification of the optimal stimulation location. ${ }^{21}$ The undesired eversion could be caused by the higher stimulation intensities, which affect larger areas, depolarizing a larger amount of fibers of the superficial peroneal nerve, leading to increased activation of the peroneal muscle, which everts the ankle joint. Patients needing higher stimulation intensities (e.g. patients with severe foot drop, primarily caused by spasticity of the calf muscles) might therefore not be candidates for training with LokoFET or FES in general. Unfortunately, this study did not asses the spasticity or the passive mechanical resistance of the ankle joint of the patients before inclusion and therefore, no relation between the degree of foot-drop, the level of spasticity, and the stimulation amplitude could be made. The therapists also mentioned that time spent on preparation instead of training could result problematic for some patients who can easily get fatigued. Post-stroke fatigue is a common stroke symptom. ${ }^{22}$ Using the abovementioned electrode arrays, might help reducing the time needed for preparation. However, the preparation time for Lokomat was far greater than that for FES, whereby the patient was placed in sitting position for the latter preparation procedure. A possible reduction of the Lokomat preparation time might therefore be more beneficial. The introduction of the FES element could result problematic for the therapists, especially when working with heavily motor impaired patients, due to the added complexity of operating both Lokomat and the FES system. In this study, FES was administered by a FES specialist and not by the therapists administering the EMGT therapy. Whether or not the added complexity of FES is a real problem is therefore unknown. Training of personnel in both the application of FES and EMGT therapy is therefore important in order to secure optimal muscle selectivity and time efficiency. Furthermore, care should be taken to include eligible patients based on their level of spasticity and fatigue. In conclusion, this feasibility study showed that LokoFET was able to provide ABI patients with active training of their ankle joint and resulted in improvements related to their foot drop impairment. However, the effectiveness of LokoFET compared to conventional EMGT therapy is still unknown. Therapists perceived LokoFET as a useful tool in the rehabilitation of gait, pointing towards possible acceptance of the technology. However, muscle selectivity and the added complexity related to the FES element of the treatment were assessed to be critical for LokoFET to be useful in clinical practice. 


\section{Lokomat and FES for foot drop}

Eur J Transl Myol 26 (3) 268-273

\section{Author's Contribution}

CBL, data collecting and analyzing; CBL, EGS manuscript writing; EGS, OKA, JFN, manuscript reviewing

\section{Acknowledgments}

We would like to thank the physiotherapists and patients who participated in the study. The work was supported by Aalborg University, Hjernesagen, the Oticon foundation, and the Otto Mønsted foundation.

Part of this paper was presented at the IFESS conference in La Grande-Motte, France 2016.

\section{Conflict of interests}

The authors declare no potential conflict of interests.

\section{Corresponding Author}

Erika G. Spaich, Department of Health Science and Technology, Aalborg University, Fredrik Bajers Vej 7 D-3, 9220 Aalborg, Denmark.

Email: espaich@hst.aau.dk

\section{E-mail of co-Authors}

Christian Bernt Laursen: cbl@hst.aau.dk Jørgen Feldbæk Nielsen: joerniel@rm.dk Ole Kæseler Andersen: oka@ @st.aau.dk

\section{References}

1. Basford JR, Chou LS, Kaufman KR, et al. An assessment of gait and balance deficits after traumatic brain injury. Arch Phys Med Rehabil 2003;84:343-9.

2. Ovbiagele B, Nguyen-Huynh MN. Stroke epidemiology: advancing our understanding of disease mechanism and therapy. Neurotherapeutics 2011;8:319-29.

3. Whittle M. Gait analysis - an introduction, whittle. 3 edition. Butterworth-Heinemann; 2001. 232.

4. Stewart JD. Foot drop: where, why and what to do? Pract Neurol. 2008;8:158-69.

5. Franceschini M, Massucci M, Ferrari L, et al. Effects of an ankle-foot orthosis on spatiotemporal parameters and energy cost of hemiparetic gait. Clin Rehabil 2003;17(4):368-72.

6. Mehrholz J, Elsner B, Werner C, et al. Electromechanical-assisted training for walking after stroke: updated evidence. Stroke 2013;44:e127-8.

7. Husemann B, Müller F, Krewer C, et al. Effects of locomotion training with assistance of a robotdriven gait orthosis in hemiparetic patients after stroke: A randomized controlled pilot study. Stroke 2007;38:349-54.

8. Hidler JM, Wall AE. Alterations in muscle activation patterns during robotic-assisted walking. Clin Biomech 2005;20:184-93.
9. Bosch PR, Harris JE, Wing K. Review of therapeutic electrical stimulation for dorsiflexion assist and orthotic substitution from the american congress of rehabilitation medicine stroke movement interventions subcommittee. Arch Phys Med Rehabil 2014;95:390-6.

10. Melo PL, Silva MT, Martins JM, Newman DJ. Technical developments of functional electrical stimulation to correct drop foot: Sensing, actuation and control strategies. Clin Biomech 2015;30(2):101-13.

11. Tong RK, Ng MF, Li LS. Effectiveness of Gait Training Using an Electromechanical Gait Trainer, With and Without Functional Electric Stimulation, in Subacute Stroke: A Randomized Controlled Trial. Arch Phys Med Rehabil 2006;87:1298-304.

12. Dohring ME, Daly JJ. Automatic synchronization of functional electrical stimulation and robotic assisted treadmill training. IEEE Trans Neural Syst Rehabil Eng 2008;16:310-3.

13. Spaich EG, Bøg MF, Erkocevic E, et al. Gait Orthosis Lokomat Combined with Functional Electrical Stimulation for Foot Drop Correction: A Feasibility Study. Jensen W, Andersen OK, Akay M, editors. Cham: Springer International Publishing; 2014;7:751-7.

14. Kvale S. Interviews: An Introduction to Qualitative Research Interviewing. SAGE Publications, Inc., 1996.

15. Hsieh H-F, Shannon SE. Three approaches to qualitative content analysis. Qual Health Res 2005;15:1277-88.

16. Craig P, Dieppe P, Macintyre S, Michie S, Nazareth I, Petticrew M. Developing and evaluating complex interventions: the new Medical Research Council guidance. BMJ 2008;337:a 1655.

17. Lin PY, Yang YR, Cheng SJ, Wang RY. The relation between ankle impairments and gait velocity and symmetry in people with stroke. Arch Phys Med Rehabil 2006;87:562-8.

18. Langhorne P, Bernhardt J, Kwakkel G. Stroke rehabilitation. Lancet 2011;377(9778):1693-702.

19. Murray MP, Spurr GB, Sepic SB, et al. Treadmill vs. floor walking: kinematics, electromyogram, and heart rate. J Appl Physiol (Bethesda, Md 1985). 1985;59:87-91.

20. Davis FD. A technology acceptance model for empirically testing new end-user information systems: Theory and results. Management 1985;Ph.D. Thesis 291.

21. Popović-Bijelić A, Bijelić G, Jorgovanović N, et al. Multi-field surface electrode for selective electrical stimulation. Artif Organs 2005;29:44852.

22. Barritt AW, Smithard DG. Targeting Fatigue in Stroke Patients. ISRN Neurol 2011;2011:1-6. 